Much innovation in digital sexual health has focussed on postal self-sampling for STIs and blood borne viruses. The available literature, with some exceptions, suggests these services appeal more to women, people with higher educational qualifications, and those from more affluent areas. A recent study suggests that people with mild learning disabilities find considerable barriers to this type of care. Where available, clinical outcomes of online care compare variably with traditional care pathways and there has been little robust economic evaluation.

Digital sexual health has huge potential to meet the needs of people who are willing and able to engage with online care. Much more ambitious care could be provided in seamless eClinics which are integrated with face-to-face services, giving the user and health care professional flexibility, continuity and choice. They have the potential to provide good value for money.

However, STIs, like digital literacy are socially patterned; the burden of STIs is disproportionately borne by those who already experience health inequalities and people experiencing health inequalities are less digitally and health literate. We need to ensure we create digital health systems which narrow rather than widen existing health divides, and evaluate them robustly.

I will summarise existing evidence for online sexual health care and draw out the major evidence gaps to help us develop inclusive, effective online sexual health care.

\section{PL10 USING PATHOGEN POPULATION GENOMICS TO STUDY THE EVOLUTION, SPREAD, AND ANTIBIOTIC RESISTANCE OF STIS}

Y Grad*. Harvard Chan School of Public Health, Boston, USA

\subsection{6/sextrans-2021-sti.10}

The analysis of genomes from populations of isolates can help address many questions relevant to the pathogen's biology and epidemiology and thus inform on clinical and public health practice. These questions include the origins and evolution of the pathogen, its spread through populations, and the genes and genotypes associated with a pathogen's antimicrobial resistance, virulence, and other phenotypes of interest. Here, I will discuss the application of population genomics to STIs, with a focus on Neisseria gonorrhoeae and with highlights from other sexually transmitted pathogens.

\section{PL11 HONOURING THE 'LIVED EXPERIENCE': A REFLECTION ON PARTICIPATORY PRACTICES IN RESEARCH}

B Kasadha*. University of Oxford, Oxford, UK

\subsection{6/sextrans-2021-sti.11}

This talk will reflect on community engagement and participatory practices implemented in health research and the contexts that enable and hinder this. The talk will explore what is needed to address the dichotomy between 'knowledge producers' and 'knowledge recipients' and the impact this has on partnerships and collaborating with communities in health research. Bakita Kasadha will draw on her experiences in community and academically led participatory health research with people living with HIV.

\section{PL12 THE NEW ABNORMAL: STI AND HIV DIVERGENCE AND CONVERGENCE IN A CHANGING UNIVERSE}

K Mayer*. Fenway Health/Harvard Medical School, USA

\subsection{6/sextrans-2021-sti.12}

Soon after the recognition of HIV as the cause of AIDS, its multiple interactions with STI were recognized. HIV-STI synergism was manifested in several ways. Individuals at increased risk for HIV were also at increased risk for STI, resulting in epidemiological synergism. The transmission of HIV was greatly facilitated by anogenital inflammation, ulcerations, and abnormal microflora, often due to STI, and HIV could alter susceptibility to some STI, examples of biological synergism. However, the subsequent development of highly effective antiretrovirals for HIV treatment and prevention altered these relationships. HIV-infected individuals who were virally suppressed on treatment were found to be incapable of transmitting HIV to uninfected partners, leading to the mantra that Undetectable $=$ Untransmissable, or $\mathrm{U}=\mathrm{U}$. The use of antiretroviral pre-exposure prophylaxis (PrEP) by sexually active, HIVinfected individuals limited their risk for HIV acquisition. Over the past decade, the recognition that antiretrovirals can protect against HIV transmission or acquisition has been associated with increasing detection of STI globally, i.e. unlinking epidemiological synergism. These new challenges present new opportunities, creating a greater need for the coordination and integration of HIV and STI diagnostic, therapeutic and preventive services, and the development of creative, consumerfriendly services focused on sexually active persons. Some jurisdictions have developed express clinics which use touchpad computers and rapid turn around NAAT screening to create a seamless experience for sexually active clients. Many of these centers use a 'status neutral' approach, i.e. providing 'one stop shopping' sexual health services, where HIV-positive and negative persons can access STI screening and treatment. Those whose HIV status is unknown, can be tested, and depending on the results, be triaged to antiretroviral treatment or PrEP. New concepts and models that are people- rather than pathogen-centered may ultimately result in better control of multiple co-prevalent pathogens.

\section{PL13 EFFECTIVENESS OF INTERVENTIONS WITHOUT RCTS \\ W Miller*. The Ohio State University, Columbus, Ohio, USA}

\subsection{6/sextrans-2021-sti.13}

Randomized clinical trials (RCTs) are generally considered the gold standard for the evaluation of interventions. In an RCT, one group is randomly assigned the intervention and another group serves as a comparison group. But RCTs can be subject to loss to follow-up and non-adherence, which can create challenges for interpretation. In addition, we often cannot randomize an intervention, whether for ethical or logistical reasons. What can be done in those circumstances?

In the past quarter century, the field of epidemiology has made dramatic progress in the use of causal methods to 
understand relationships between interventions and exposures (or risk factors) and disease or behavioral outcomes. In general, this approach allows many potential risk factors to be recast theoretically as an intervention. For example, a behavior such as condomless sex, which could never be assigned in an RCT, could be thought of as an 'intervention' or perhaps more appropriately, as the comparison to an intervention of sex with a condom. Once we reframe the question, several methods are available to account for potential biases that may arise in observational studies, including selection bias and confounding.

In this session, we will consider this reframing process using examples, real and hypothetical, from the fields of sexually transmitted infections and HIV. The process typically begins with a carefully constructed causal diagram, called a directed acyclic graph or DAG. DAGs are used to outline the causal pathways related to the research question. The DAG accounts for both the specific factors and temporality of the relationships. With the DAGs in hand, we can plan the appropriate analyses for the specific research questions. Without equations or complex statistics, we will consider three commonly used analytical approaches: propensity scores, instrumental variables, and marginal structural models (epidemiology's MSM). We will also briefly consider other quasi-experimental approaches for policy evaluations.

\section{PL14 WHAT'S TO KNOW AND WHAT'S TO BE DONE - IMPLEMENTING AND MONITORING STI AND HIV PROGRAMMES}

Y Pillay*. Clinton Health Access Initiative, Pretoria, South Africa

10.1136/sextrans-2021-sti.14

With decades of experience implementing STI and HIV programmes globally what do we know and what are we still learning? Implementation science is a relatively new approach to understanding what facilitates rapid implementation and the barriers to implementation.

With a rising tide of some STIs and need to eliminate HIV as a public health threat, this presentation will focus on experiences of a national programme manager implementing STI, HIV and other programmes in an upper middle-income country - South Africa. Looking ahead does the COVID-19 pandemic provide us with opportunities to do things differently and if yes, what can they be?

\section{PL15 DEVELOPMENT OF IMMUNOTHERAPEUTIC APPROACHES AGAINST GONORRHEA}

S Ram* , S Gulati, J Shaughnessy, P Rice. University of Massachusetts Medical School, Worcester, USA

10.1136/sextrans-2021-sti.15

Multidrug-resistant (MDR) Neisseria gonorrhoeae $(\mathrm{Ng})$ is a global health problem. Targeting virulence factors can circumvent the ability of gonococci to resist conventional antimicrobials. Resistance to such agents, if it were to occur, would result loss of bacterial fitness; attenuated 'escape mutants' would be susceptible to host immune defenses. A unique gonococcal immune evasion strategy involves capping of lipooligosaccharide (LOS) with sialic acid by gonococcal sialyltransferase (Lst), utilizing host-derived CMP-sialic acid (CMP-Neu5Ac in humans). LOS Neu5Ac renders gonococci resistant to complement (by binding the complement inhibitor, factor $\mathrm{H}(\mathrm{FH})$ ) and cationic antimicrobial peptides (CAMPs). LOS sialylation is important for gonococcal virulence in humans and in experimental mouse models. A chimeric protein that fuses the gonococcal binding domains of human FH (lacks complement-inhibiting activity) with human $\operatorname{IgG} 1 \mathrm{Fc}(\mathrm{FH} / \mathrm{Fc})$ enhances complement activation on the bacterium and mediates complement-dependent killing of a wide array of gonococcal isolates in vitro. Intravaginal administration of $\mathrm{FH} / \mathrm{Fc}$ attenuates bacterial burden in the mouse vaginal colonization model. Gonococcal Lst has broad substrate specificity and can utilize CMP salts of sialic acid analogs, such as legionaminic (CMP-Leg) or ketodeoxynonulosonic (CMP-Kdn). Incorporation of Leg or $\mathrm{Kdn}$ into LOS substitutes for the Neu5Ac sialic acid cap and restores bacterial susceptibility to complement and CAMPs. Intravaginal CMP-Leg or CMP-Kdn administration mediates CAMPdependent clearance of MDR Ng in mice. An LOS epitope that is recognized by monoclonal antibody $(\mathrm{mAb}) 2 \mathrm{C} 7$ is expressed by $\sim 95 \%$ of $\mathrm{Ng}$ isolates in vivo, can also be sialylated and is critical for virulence. The 2C7 epitope has also been fashioned as a vaccine candidate and is pending a human trial. mAb $2 \mathrm{C} 7$ delivered either intravaginally or systemically, or as a DNA-encoded mAb (passive vaccination) clears gonococci from mouse vaginas in a complementdependent manner. In conclusion, targeting LOS-related virulence mechanisms is an innovate approach to combat MDR Ng.

\section{PL16 STRENGTHENING COMMUNITIES' RESPONSE, STAKE, AND ENGAGEMENT IN HIV/STI RESEARCH}

S Rawat*. The Humsafar Trust, Mumbai, India

\subsection{6/sextrans-2021-sti.16}

Though HIV interventions have been implemented in India since over a decade, HIV prevalence among men who have sex with men (MSM) and transgender women (TGW) in India $(2.7 \%$ and $3.1 \%$, respectively) continues to be $10-15$ times higher than that observed among heterosexual communities $(0.34 \%)$. While evidence for nationwide prevalence of STIs is lacking, independent studies have estimated STI rates at $12-38 \%$. Further, clinic data from The Humsafar Trust-an LGBTQ+ organization in Mumbai providing HIV outreach services to over $7500 \mathrm{MSM}$ and TGW annuallyhas estimated syphilis rates to be $10 \%$ in 2018 and $12 \%$ in 2019. A key challenge in addressing these STI trends is the limited availability of prevalence-focused programmatic implementation and bio-behavioral research on STI awareness/health seeking behavior among vulnerable communities. Though syphilis testing has been integrated in national HIV interventions, management of other STIs is syndromic resulting in under-diagnoses and under-reporting. Further, lack of dedicated resources toward building knowledge and awareness of STIs among vulnerable communities leads to low risk-perception and compromised uptake of testing and treatment. While evidence building and documentation can 